\title{
Cartographie De La Salinite A La Surface Du Sol Dans Une Zone Du Prerif. Cas De La Region De L'ouergha
}

\author{
Mohamed Sadiki \\ Laboratoire de Géosciences Appliquées, \\ Département de Géologie, Université Ibn Tofail Kenitra \\ Amal Markhi \\ Laboratoire GEOHYD, Département de Géologie, \\ Université Kadi Ayyad Marrakech
}

Hicham Elbelrhiti

Département des Sciences Fondamentales et Appliquées (DSFA), Institut Agronomique et Vétérinaire Hassan II, Madinat Alirfan, Rabat, Morocco

Souad Mrabet

Laboratoire de Géosciences Appliquées, Département de Géologie, Université Ibn Tofail Kenitra

doi: 10.19044/esj.2016.v12n3p197 URL:http://dx.doi.org/10.19044/esj.2016.v12n3p197

\begin{abstract}
The soil and groundwater salinization phenomenon in semi-arid to arid climate is considered as a real threat to safety and food quality. There are several factors that present soil salinity, some factors are purely climatic (temperature, rainfall levels, lack of drainage, composition of the rock) or human-induced (using salt water to irrigation). The aim of the work is to take stock of the surface condition at a specified scale of soil salinity by taking satellite images Landsat TM 2009 and ASTER 2003 with 15 m and 30 m of resolution respectively. This study allows us to detect the potential of remote sensing data to see a set of thematic maps that distinguish, evaluate and locate their extended saline soils on the surface of the study area. The methods of satellite image processing are for understanding of soil salinization process, assess their extensive and locate areas vulnerable to soil and water salinization. Evaluation of the results of applying this method on Landsat TM gave an accuracy of $87 \%$. This study also allows us to highlight spectral indices that again demonstrate the natural origin, related to the lithology of groundwater salinity in the study area. These various indices largely exploit the difference spectral response of vegetation and soils in the red band (R) and near infrared band (PIR) which is related to the density of
\end{abstract}


green vegetation the NDSI and NDVI which allows a very good distinction between areas of salinity and vegetation area.

Keywords: Soil salinity, remote sensing, l’Ouergha region, Morocco

\section{Résumé}

Le phénomène de la salinisation des sols et des nappes dans zones à climat aride à semi-aride est considéré comme une menace réelle pour la sécurité et la qualité alimentaire. Il y a plusieurs facteurs qui mettent en évidence la salinité des sols, à savoir des facteurs purement climatiques (Température, niveau des précipitations, absence de drainage, composition de la roche mère) ou anthropiques (utilisation de l'eau salée pour l'irrigation). Le but du travail est d'établir un bilan de l'état de surface à une échelle déterminée de la salinité des sols en prélevant des images satellitaires Landsat TM 2009 et ASTER 2003, de $15 \mathrm{~m}$ et $30 \mathrm{~m}$ de résolution respectivement. Cette étude a permis de détecter les potentialités offertes par les données de télédétection pour voir un ensemble de cartes thématiques qui distinguent, localisent et évaluent les étendues des sols salins à la surface de la zone d'étude dans la région de l'Ouergha. Les méthodes de traitement d'image satellitaire ont permis de comprendre les processus de salinisation des sols, d'évaluer leurs étendues et localiser les zones vulnérables à la salinisation des sols et de l'eau. L'évaluation des résultats de l'application de cette méthode sur l'image Landsat TM a donné une précision de 87 \%. Cette étude nous a permis également de mettre en évidence des indices spectraux qui démontrent encore une fois l’origine naturelle, liée à la lithologie de la salinité des eaux souterraines de la région étudiée. Ces différents indices exploitent essentiellement la différence de réponse spectrale de la végétation et des sols dans la bande rouge $(\mathrm{R})$ et la bande proche infrarouge (PIR), il s'agit de NDSI et NDVI qui ont permis une parfaite distinction entre les zones de salinité et les zones de végétation.

Mots clés : Cartographie de la salinité, télédétection, la région de l’Ouergha, Maroc

\section{Introduction}

La région de l’Ouergha est dotée d'une eau souterraine qui est jugée de qualité impropre à la consommation. En effet la population locale souffre du problème de la salinité des eaux et de son effet néfaste sur la santé, l’agriculture et l'élevage.

Le bassin de l'Ouergha est situé sur des terrains de lithologie variée qui appartenant à la zone du prérif. Du point de vue hydrogéologique, les 
points d'eau sont nombreux du fait de l'existence de plusieurs poches d'eau mais assez souvent salés (Thauvin, 1971).

La région étudiée est située au nord-ouest du Maroc et délimitée par les latitudes 34.4 et 34.7 vers le nord, et par les longitudes 5.1 et 5.8 vers l'ouest. Le secteur d'étude est compris entre la région de Mjaâra où est édifié le barrage Al Wahda et la région de Khenichet près de la confluence SebouOuergha (Fig. 1).

La zone d'étude est située dans la zone externe de la chaine rifaine, comblée par d'épaisses séries mésozoïques et cénozoïques (Piquee et al., 2007). C’est une zone structurale du prérif dont la lithologie comporte essentiellement des faciès marneux. Dans le Prérif interne, des terrains jurassiques sont conservés mais le Prérif externe, par contre, ne montre qu'un olistostrome (complexe prérifain ou nappe prérifaine) réalisant le comblement, au Miocène moyen et supérieur, de l'avant fosse rifaine. Les formes topographiques de cette zone, à substratum notamment marneux, montrent une morphologie nettement pénéplanée par comparaison aux autres zones à formations généralement compétentes (calcaires, grès schisteux, etc.).

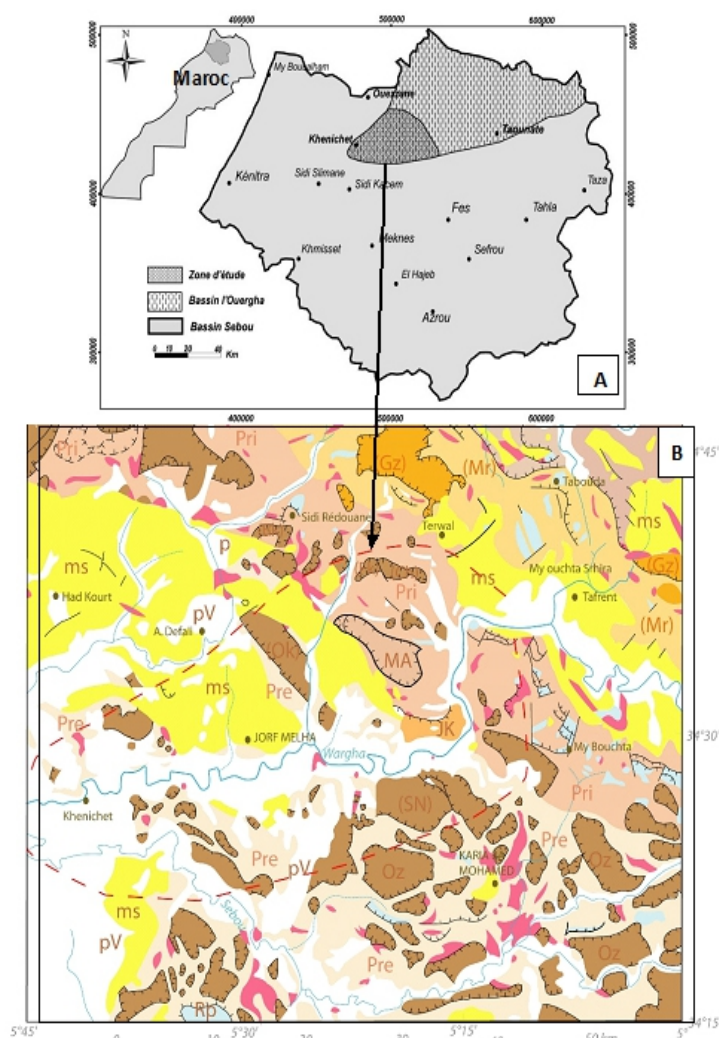

Figure 1: A : Situation de la zone d'étude dans le bassin hydrologique du Sebou. B : Carte structurale de la région étudiée : Rides prérifaines (Rp), prérif externe (Pre), prérif interne (Pri), Mesorif (Mr), Nappes d'origine intrarifaine (JK, MA, LK, Tg et Oz), Miocène post-nappes (ms), Pliocène (P), Villafranchien (pV) et Quaternaire (q). 
Le bassin versant de l’Ouergha est un sous bassin du grand bassin de Sebou, il s'étend sur environ $7300 \mathrm{~km}^{2}$ de superficie. Les niveaux géologiques susceptibles de former des aquifères de quelques importances sont peu nombreux dans le Prérif (Thauvin, 1971). Ce sont les calcaires et les dolomies du Lias, la série marno-gréseuse post-Eocène de la nappe d'Ouezzane, les grés et les sables du Tortonien inférieur et du Miocène supérieur-Pliocène, les sédiments caillouteux du Plio-Villafranchien et le Quaternaire graveleux des fonds des oueds et des terrasses.

L'étude hydrochimique et statistique des eaux ont permis de démontrer la relation entre les lithofacies évaporitiques qui sont souvent en état d’affleurement et la salinité des eaux dans la région.

Dans le but de faire une cartographie générale de ces facies à l'affleurement nous avons utilisé la télédétection pour repérer la salinité à la surface du sol. Ceci a été prouvée par beaucoup d'auteurs et qui ont montré que la télédétection, particulièrement dans la bande spectrale du visible, rapporte des informations spatiales fortement corrélées avec la salinité (Mougenot, 1993; Rahman et al., 1994; Khan et al., 2001; Metternicht and Zinck, 1997, 2003, Douaoui, Nicolas, and Walter 2006) ou les caractéristiques de la surface du sol (Metternicht and Zinck, 1998). De plus, plusieurs auteurs ont démontré l'avantage de la combinaison des données de la télédétection avec les informations observées et recueillies sur la surface de la terre (Bishop and McBratney, 2001; Carré and Girard, 2002 ; Wannakomol, 2005).

C’est dans ce contexte que la méthodologie utilisée pour la production des cartes de salinité à la surface du sol à moyenne échelle de notre région se base sur des données satellitaires (Landsat TM 2009 à 30 m de résolution et ASTER 2003 à 15 m de résolution) et sur les données in situ. Elle comprend une analyse des indices de salinité basés sur la végétation et la luminosité qui indiquent le degré de l’influence de la salinité sur l'humidité des plantes (Vincent et al. 1996), et une classification de la surface du sol (supervisée et non supervisée). Les fausses compositions de couleur qui ont été utilisées comme base de traitement dans le cadre de cette étude sont les bandes 4, 3 et 2 et les bandes 3, 2 et 1 comme rouge, vert et bleu pour les images Landsat et ASTER respectivement (Fig. 2 et Fig. 3). 


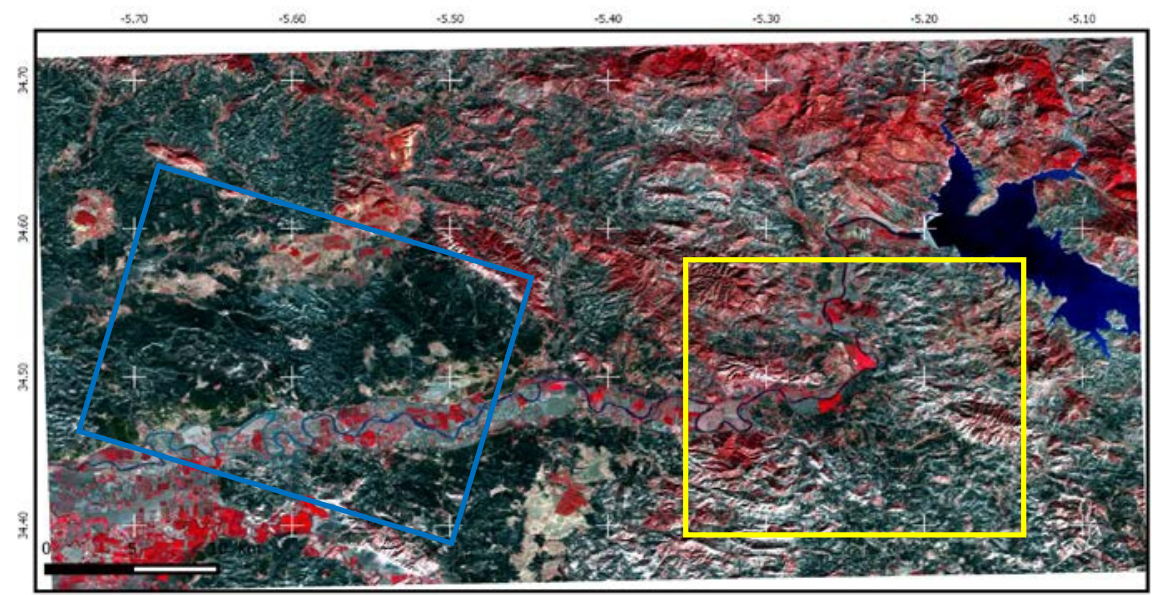

Figure 2 : Composition colorée de l'image Landsat TM 11/11/2009. Les bandes 4, 3 et 2 comme rouge, vert et bleu ont été utilisées comme base pour la classification et le calcul des indices. Carreaux jaune et bleu représentent l'emplacement des images ASTER désormais baptisées amont et aval
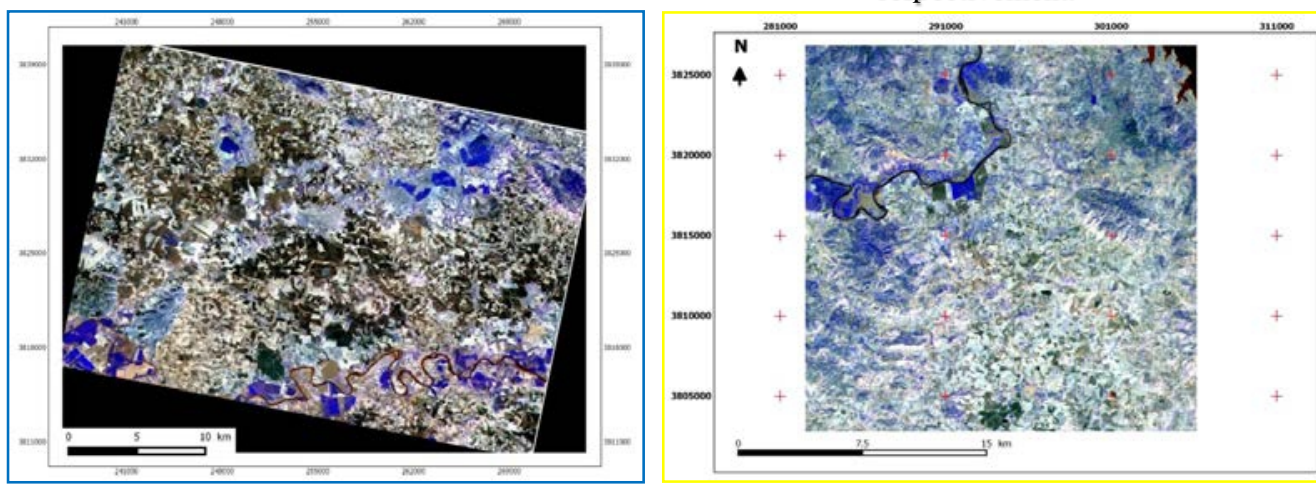

Figure 3: Composition colorée des images ASTER 2003. Les bandes 3, 2 et 1 comme rouge, vert et bleu ont été utilisées. A droite c'est l'image ASTER amont, et à gauche c'est l'image ASTER aval.

\section{Matériels et méthodes utilisés}

Les images Satellitaires ont constitué la source d'information majeure pour réaliser la base cartographique nécessaire et aussi pour connaitre l'évolution dans l'espace des composantes de la surface du bassin étudié. Pour réaliser cette étude, nous nous sommes proposé de combiner plusieurs informations géographiques intimement liées à l'espace dont elles doivent donner la représentation la plus appropriée.

Les données de télédétection utilisées dans le cadre de ces études ont été prises par différents systèmes d'observation liés à des satellites. Les images TM étaient fournies gratuitement par la plateforme GLCF (The Global Land Cover Facility) mis en place par l'université de Maryland sur son site officiel. 
Dans cette étude, nous avons utilisé deux scènes ASTER, choisies sur la base des résultats des traitements des images Landsat. Elles se sont utilisées pour nous donner plus de précision vue leurs capacités spectrales. Les deux images sont prises en Juin 2003 (USGS, and Japan ASTER Program, 2003).

Les données sont de type :

- Landsat 5 (Thematic Mapper, TM): 11 Novembre 2009 Path/Row 201/036.

- ASTER (Advanced Space borne Thermal Emission and Reflection Radiometer).

- Un modèle numérique d'altitude SRTM (30 m), décrivant la géomorphologie du bassin versant.

Le but du traitement d'images satellitaires est d'extraire de celles-ci des informations utiles pour la compréhension du phénomène de la salinisation des eaux souterraines ainsi que de la surface du sol dans le bassin de l'Ouergha.

Le traitement et les classifications des images Landsat et ASTER ont été faits à l'aide du programme ENVI. La mise en page finale des cartes ainsi que le calcule d'indices ont été fait par le logiciel QGIS.

Le traitement des images TM et ASTER s'est déroulé en 4 phases: prétraitement, rehaussement de l'image, transformation de l'image, classification et analyse de l'image (Abourida, 2007). La méthode retenue pour la classification de l'image est celle de Maximum vraisemblance (Maximum Likelihood). Elle se base sur un modèle radiométrique correct et adapté à la région étudiée. Elle permet de ne classer que ce qui correspond au but recherché et de mettre dans une classe toutes les autres composantes de l'image qui ne sont pas utiles (Girard et Girard, 1999).

Pour détecter la salinité à la surface du sol, nous avons calculé un certain nombre d'indices mathématiques sur les images satellitaires TM et ASTER. Ces indices résultent de la combinaison de plusieurs bandes du même spectre (voir les formules ci-après) et qui ont été développés par Abbas et Khan (Abbas et Khan, 2007) et fondés sur l'hypothèse que cette transformation rendrait l'information demandée de salinité plus proéminente tout en supprimant les effets d'autres utilisation et occupations des sols (Abbas et al., 2013). Des approche semblables sont rapportées dans la littérature et qui ont été employées dans des régions affectées par le sel, on cite (Dwivedi 1992; Srivastaka et al., 1997; Al Khair, 2003 ; Khan et al., 2005).

Les indices utilisés dans le cadre de cette étude sont :

- Pour l'image Landsat nous avons calculé les indices suivants :

Indice de Salinité (SI) :

$$
S I=\sqrt{B 1 * B 3}
$$


Indice de Salinité par Différence Normalisée (NDSI) :

$$
N D S I=(B 3-B 4) /(B 3+B 4)
$$

Indice de Luminosité (BI) :

$$
B I=\sqrt{B 3+B 4}
$$

Indice de Végétation par Différence Normalisée :

$$
N D V I=(B 4-B 3) /(B 3+B 4)
$$

Dont B1, B3 et B4 sont respectivement les bandes 1,3 et 4 de l'image Landsat.

- Pour les images ASTER, les indices utilisés sont comme suit :

$$
\begin{aligned}
& N D S I=(B 2-B 3) /(B 2+B 3) \\
& N D V I=(B 3-B 2) /(B 2+B 3)
\end{aligned}
$$

Dont B2 et B3 sont respectivement les bandes 2 et 3 de l'image ASTER.

La figure 4 montre la démarche méthodologique utilisée dans le cadre de ce travail pour cartographier la salinité à la surface du sol. Les résultats de l'analyse des indices ainsi que ceux de la classification et leurs interprétations sont indiqués dans la partie résultats et discussion.

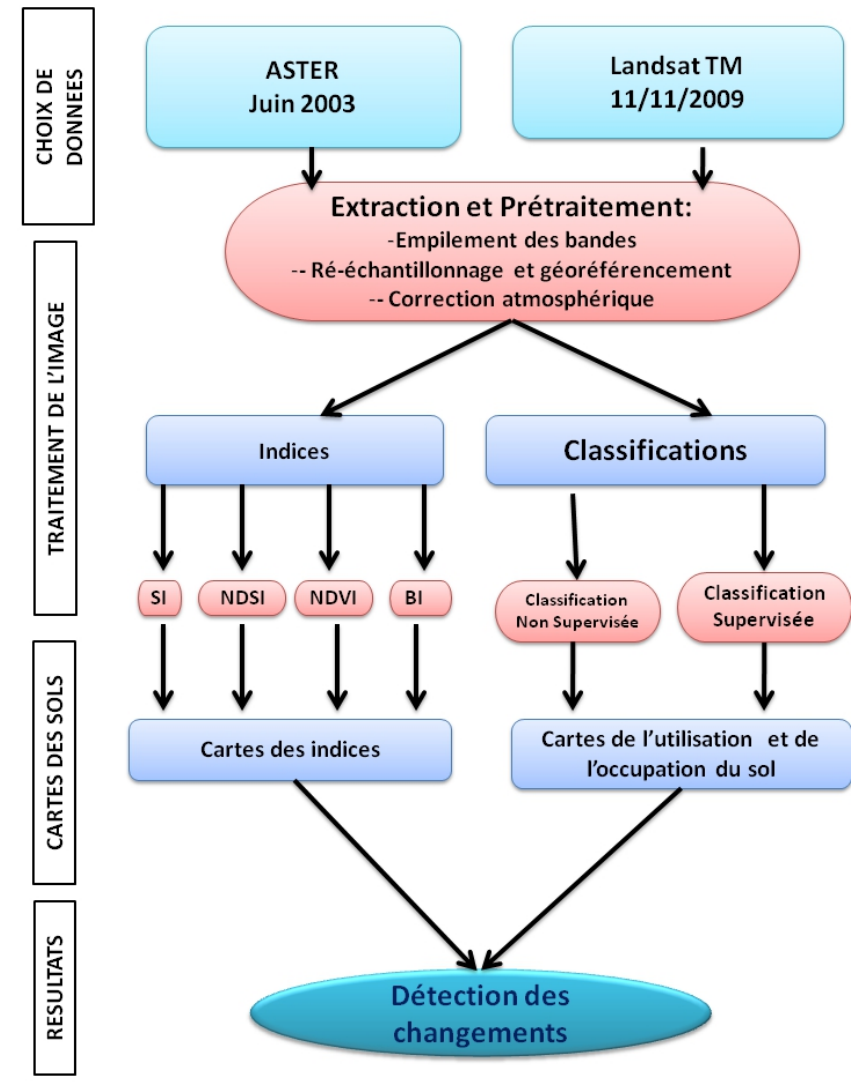

Figure 4 : Démarche méthodologique pour cartographier la salinité à la surface du sol. 


\section{Résultats et discussions}

\section{Résultats de l'analyse des eaux souterraines}

La mesure de la conductivité des eaux souterraines montre que celleci varie entre $450 \mu \mathrm{S} / \mathrm{cm}$ et $9050 \mu \mathrm{S} / \mathrm{cm}$ dans l'espace d'étude. La figure 5 montre que la conductivité augmente dans le sens d'écoulement des eaux souterraines (en général de l'est vers l'ouest du bassin versant). Ainsi, elle permet de constater que les eaux souterraines du bassin s'enrichissent en minéralisation durant leur écoulement. Les valeurs d'analyses de TDS soutiennent cet enrichissement. Elles varient spatialement de la même façon que celle de la conductivité électrique (Fig. 6). La concentration en matière solide dissoute totale des eaux souterraines varie entre $200 \mathrm{mg} / \mathrm{l}$ et 5500 mg/l. Ceci est lié (Sadiki et Mrabet, 2015):

- Au lessivage des formations traversées et en particulier des terrains évaporitiques;

- A l'évaporation: lorsque le niveau piézométrique est proche de la surface du sol (Dib et al., 2010).

La projection spatiale des données physico-chimiques des eaux s'avèrerait être d'une grande utilité dans la caractérisation physico-chimique des eaux. Elle montre que celles-ci pourraient acquérir au contact des terrains qu'elles rencontrent divers éléments qui vont influer sur leur qualité. Ainsi l'eau à l'état naturel peut contenir: des matières dissoutes provenant des terrains traversés (calcium, magnésium, sodium, potassium, bicarbonates, sulfates, chlorures,...), des particules en suspension et des éléments d'origine anthropique. Donc la qualité des eaux est dépendante d'un certain nombre de paramètres, dont la quantité de ces paramètres va définir l'état de la qualité de l'eau.

Nous avons pu déterminer à partir des analyses faites sur toute la région, deux zones bien distinctes; une zone en amont, avec une prédominance des bicarbonates et des ions calcium et magnésium caractérisant une eau de faible salinité. Et une zone en aval du bassin, avec une salinité importante liée aux $\mathrm{NaCl}$ et aux sulfates (Sadiki et Mrabet, 2015): 


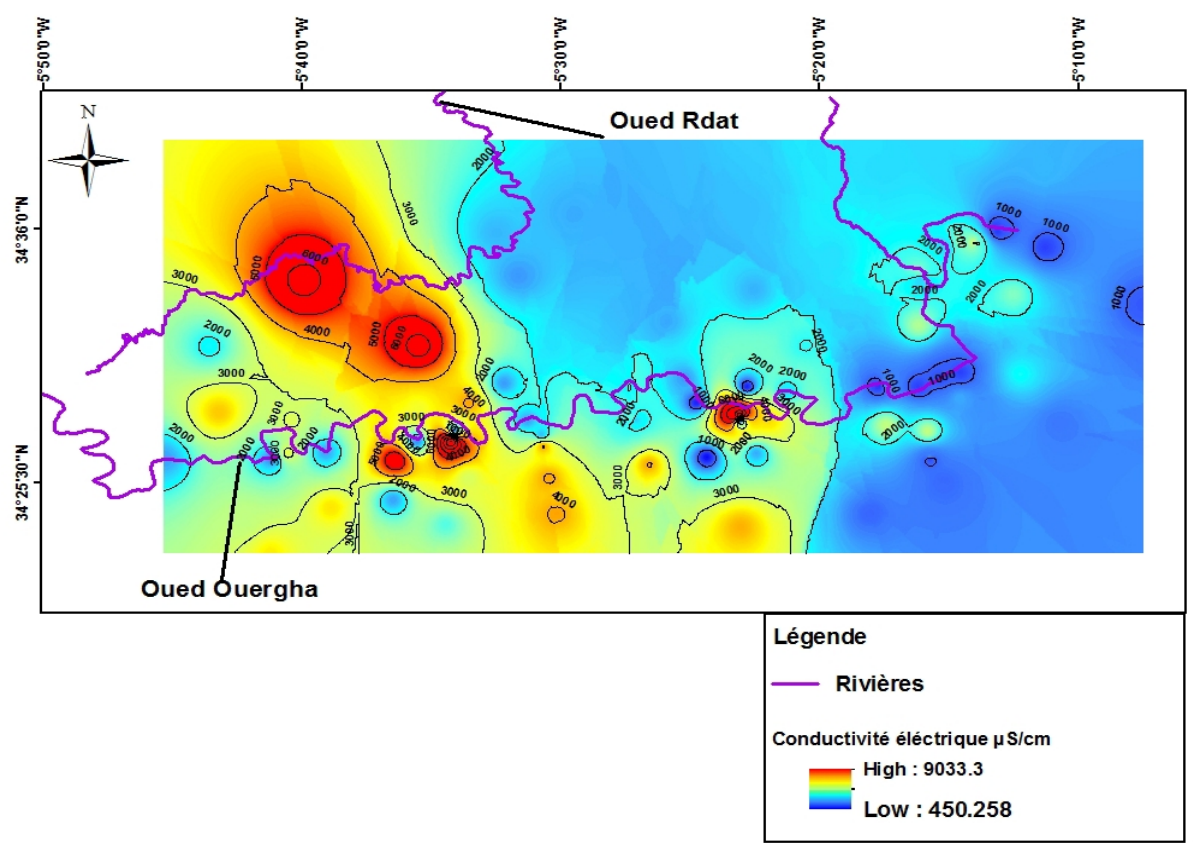

Figure 5: Variation spatiale des valeurs de la conductivité électrique $(\mu \mathrm{S} / \mathrm{cm})$ des eaux souterraines.

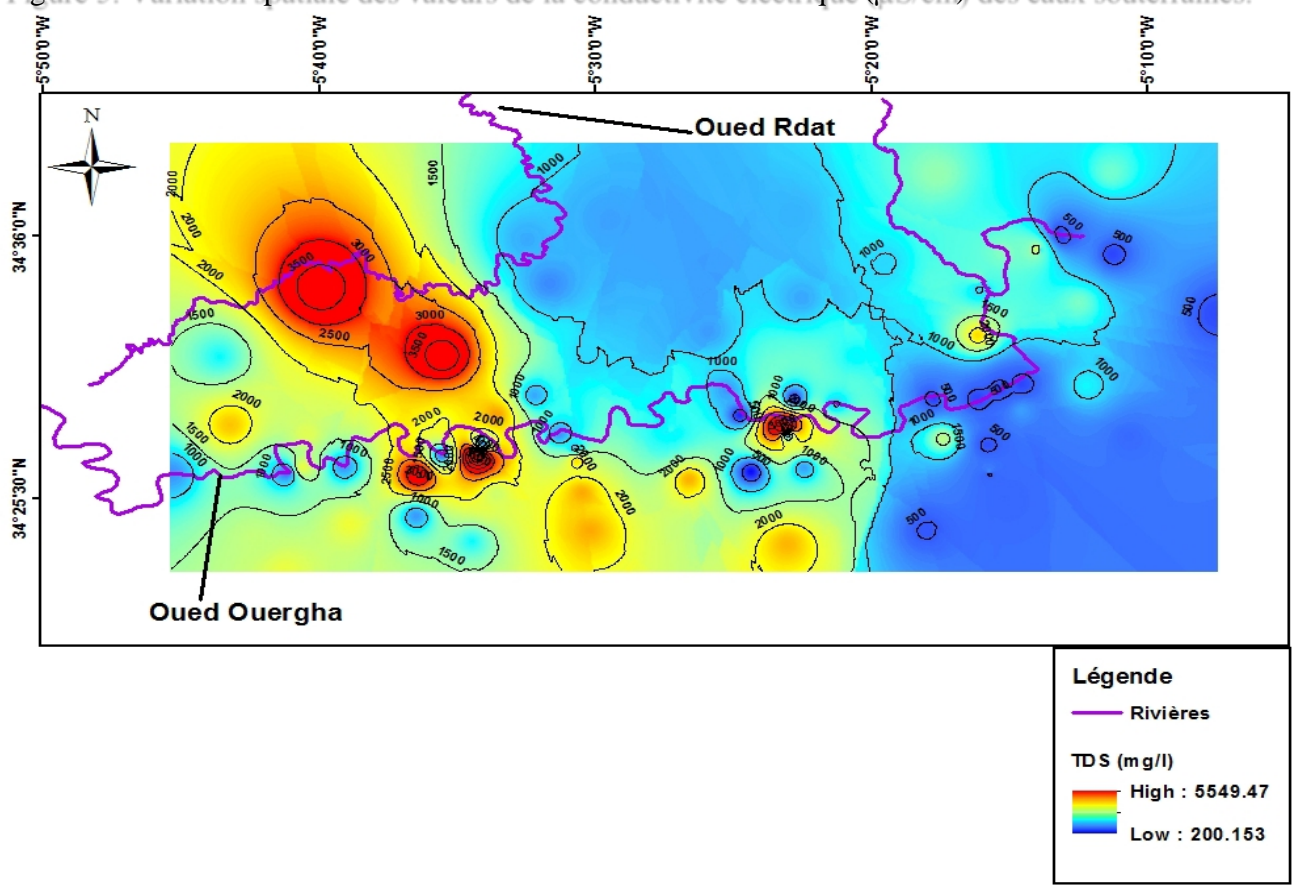

Figure 6: Variation spatiale des valeurs de TDS (mg/l) des eaux souterraines. 


\section{Résultats de traitements numériques des images satellitaires Classification non supervisée}

Indépendamment de l'interprétation visuelle sur l'imagerie satellitaire pour déterminer des secteurs affectés par la salinité, les images satellites ont été également classifiées en utilisant le logiciel ENVI, par une classification non supervisée de type IsoData.

Les résultats de la classification non supervisée (Isodata) des images Landsat TM et les images ASTER sont représentés dans la figure 7. A partir des résultats de cette classification, nous pouvons constater clairement que ce type de traitement peut donner une idée générale sur les classes de l'utilisation du sol dans la région d'étude. En effet, sept classes ont été révélées par la méthode Isodata.

En se basant sur les résultats de la classification non supervisée et sur les données de terrain, nous pouvons distinguer les classes suivantes :

Les classes de l'utilisation du sol à partir des images Landsat TM :

- Classe 1 représente les ressources en eau de la région.

- Classes 2, 3 et 4 représentent les plantes naturelles et implantées et les arbres et forets.

- Classe 5 et 6 représentent les terres nus et les socles.

- Classe 7 représente les zones affectées par la salinité.

Les classes de l'utilisation du sol à partir des images ASTER :

- Les classes 1, 2 et 3 représentent les ressources en eau, les plantes irriguées et des arbres et forets.

- Les classes 4,5 et 6 représentent la végétation saisonnière, les cultures non irriguées.

- $\quad$ La classe 7 représente la zone affectée par la salinité.

L’analyse des statistiques de résultats des classifications non supervisées montre une infériorité des zones affectées par la salinité, dans toute la région d'étude (Landsat), par rapport aux autres classes d'utilisation de sols (Tab.

1). Les statistiques de classes appliquées sur l’image Landsat révèlent un pourcentage de zone de salinité qui ne dépasse pas 9\% pour la méthode Isodata. En revanche, les statistiques appliquées sur les résultats de la classification non supervisée des images ASTER, qui ciblent des zones soupçonnées de salinité, ont montré une prédominance de 

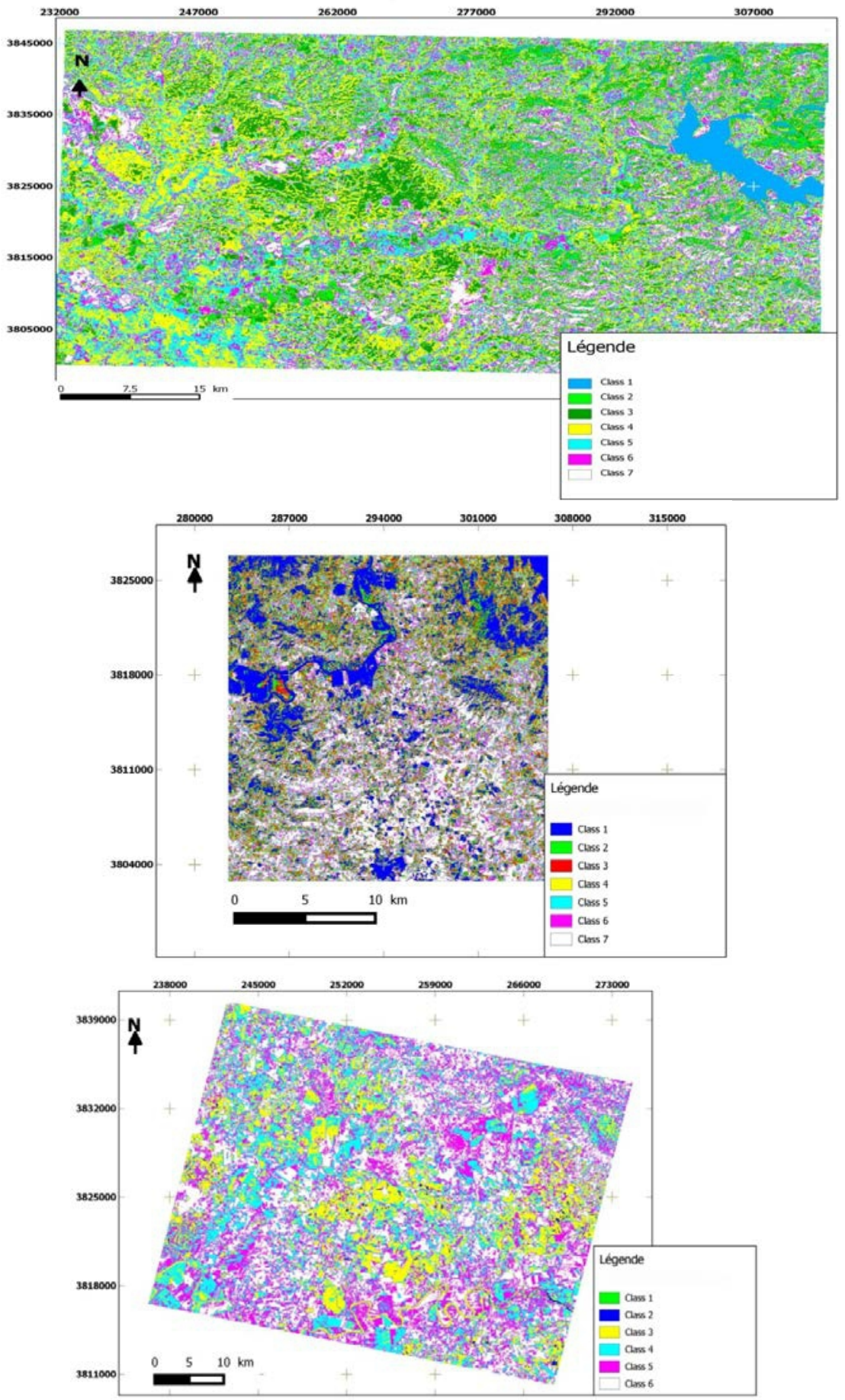

Figure 7 : Résultats de la classification non supervisée d'IsoData

la classe de salinité par rapports aux six autres classes. Cette prédominance est traduite par des pourcentages élevés de $20 \%$ et $22 \%$, selon la méthode de type Iso-Data, pour les images ASTER amont et ASTER aval respectivement. 


\begin{tabular}{|c|c|c|c|}
\hline Image & Classes & Superficie $^{2}$ & Pourcentage \% \\
\hline \multirow{5}{*}{ Landsat TM 2009 } & Classe 1 & 325791056.1051 & 8.035 \\
\cline { 2 - 4 } & Classe 2 & 135525521.3800 & 3.342 \\
\cline { 2 - 4 } & Classe 3 & 591237935.9221 & 14.581 \\
\cline { 2 - 4 } & Class e 4 & 1184479419.6884 & 29.212 \\
\cline { 2 - 4 } & Classe 5 & 944581224.9517 & 23.295 \\
\cline { 2 - 4 } & Classe 6 & 513625234.7112 & 12.667 \\
\cline { 2 - 4 } ASTER amont & Classe 7 & 359579127.8271 & 8.868 \\
\cline { 2 - 4 } & Classe 1 & 104980174.1962 & 18.491 \\
\cline { 2 - 4 } & Classe 2 & 64269158.5917 & 11.320 \\
\cline { 2 - 4 } & Classe 3 & 74513194.7941 & 13.124 \\
\cline { 2 - 4 } & Class e 4 & 74194456.5536 & 13.068 \\
\cline { 2 - 4 } & Classe 5 & 69189750.2749 & 12.187 \\
\cline { 2 - 4 } & Classe 6 & 65110080.2410 & 20.341 \\
\hline & Classe 7 & 115485750.7141 & 30.572 \\
\hline & Classe 1 & 354092610.4495 & 0.383 \\
\cline { 2 - 4 } & Classe 2 & 4432516.8483 & 10.770 \\
\cline { 2 - 4 } & Classe 3 & 124737220.7936 & 16.782 \\
\hline & Class e 4 & 194366872.2594 & 18.831 \\
\hline & Classe 5 & 218106091.8865 & 22.662 \\
\hline
\end{tabular}

Tableau 1 : Statistiques des classes à partir de la méthode de type IsoData :

En général, par rapport aux résultats de la classification non supervisée des images Landsat TM, les résultats issus de la classification non supervisée de type isodata des images ASTER permettent une différentiation plus nette entre les classes, surtout celles qui sont salines de celles qui ne le sont pas.

Cette prédominance est traduite par des pourcentages élevés de la classe de salinité.

\section{Classification supervisée}

La connaissance à priori du terrain, a permis de choisir également la classification supervisée. Elle consiste à classer les données vis à vis de zones d'apprentissage choisis par l'utilisateur. La méthode utilisée dans cette étude est une procédure supervisée, appelée classification par maximum de vraisemblance (Richards and Xiuping, 1999). Cette méthode est considérée comme une technique puissante de classification selon plusieurs auteurs (Escadafal et al., 1988; 1989; 1993; 1994; Boulahouat \& Naert, 1996; Gilliot \& Girard, 1997; Bonn, 1998; Hamdi-Aïssa, 2001 ; Schowengerdt, 2007).

Les résultats de la classification supervisée de type maximum vraisemblance appliquée sur les images Landsat et ASTER sont représentés dans la figure 8. Les classes dégagées de cette classification sont les suivantes :

- Classes 1 et 2 représentent les cultures irriguées, les cultures non irriguées et la végétation naturelles. 
- Classe 3 représente les ressources en eau de la région.

- Classe 4 représente les roches carbonatées et gypsifères.

- Classe 5 représente l'habitation de la région.

- Classe 6 représente les arbres fruitiers et les forêts.

- Classe 7 représente les sols nus et le socle.

L'analyse de ces résultats permet de révéler plusieurs points intéressants sur le couplage des données Landsat TM et ASTER dans l'étude de la cartographie de l'utilisation des sols dans la région de l'Ouergha. Ces points peuvent être résumés comme suit:

- La méthode de classification supervisée de type maximum vraisemblance permet de cartographier sept classes de l'utilisation du sol dans la région de l'Ouergha sans laisser aucune zone sans classification. Ceci est valable que ce soit pour l'image Landsat TM ou pour les images ASTER. Ceci nous permet de dire que notre zone d'étude est bio-physiquement homogène, et que toutes les classes sont bien représentées dans toute la zone d'étude.

- Les classes de cultures et la classe des ressources en eau sont très nettement distinctes par rapport aux autres classes. Elles sont similaires à la fois pour Landsat TM et ASTER.

- La détection de la classe des arbres fruitiers et des forêts dans l'image Landsat est plus fiable que celle dans l'image ASTER. Cela est dû normalement au fait qu'il a plus d'échantillons de cette classe dans l'image Landsat TM que dans celle d'ASTER.

- Les pourcentages des domaines affectés par la salinité révélés à partir de la classification supervisée sont inférieurs que ceux des domaines affectés par la salinité révélés à partir de la classification non supervisée. Ceci est dû au fait, qu'au cours de la classification supervisée, on a classé les sols nus et ceux qui sont salins en deux zones d'intérêt différentes.

- La classification supervisée montre une prédominance de zone de salinité dans la région de l'Ouergha, ceci est confirmé par le fait qu'il y a un pourcentage faible des zones de cultures irriguées malgré l'importance des ressource en eau permanentes de la région manifestées par la ravière de l'Ouergha et ces affluents ainsi que le barrage d'Alwahda en amont.

- Le couvert végétal de la région est caractérisé par une végétation basée essentiellement sur des cultures bour et une végétation annuelle liée aux climats des saisons de l'année. L'arboriculture dans la région de l'Ouergha est moins développée et basée surtout sur l'olivier dans les régions de grandes altitudes et de l'eucalyptus sous forme de forêts éparses. 
Le tableau 2 montre les différentes classes issues de la classification supervisée des images Landsat TM et ASTER, ainsi que la superficie qu'occupent chaque classe, et par conséquent le pourcentage de chaque classe dans le résultat de la classification de chaque type d'image.

Dans les vérifications sur le terrain menées dans le mois du juillet 2013, à partir de 30 sites d'investigation sélectionnés et qui devraient être affectés par le sel selon les résultats de classification supervisée, 26 sites ont montré des roches et / ou des sols salés et gypsifères à la surface, et le reste de 4 sites ont aussi un potentiel pour la salinité vu qu'ils abritent des sédiments d'origine évaporitiques. Par conséquent, l'exactitude de cette classification supervisée pour définir la zone affectée de sel est de 87 pour cent. Bien que la précision de cette technique de classification est satisfaisante, les sites d'investigation sélectionnés ne pourraient pas représentés tous les sites de la région, étant donné qu'il y a des zones de sol nu et qui présentent des indices de salinité tandis qu'elles montrent des signes de réflectance différents de ceux des zones de sels proprement dites. Par conséquent, la technique de calcul mathématique des indices radiométriques a été utilisée pour palier à l'élimination ou à la réduction de ces problèmes.

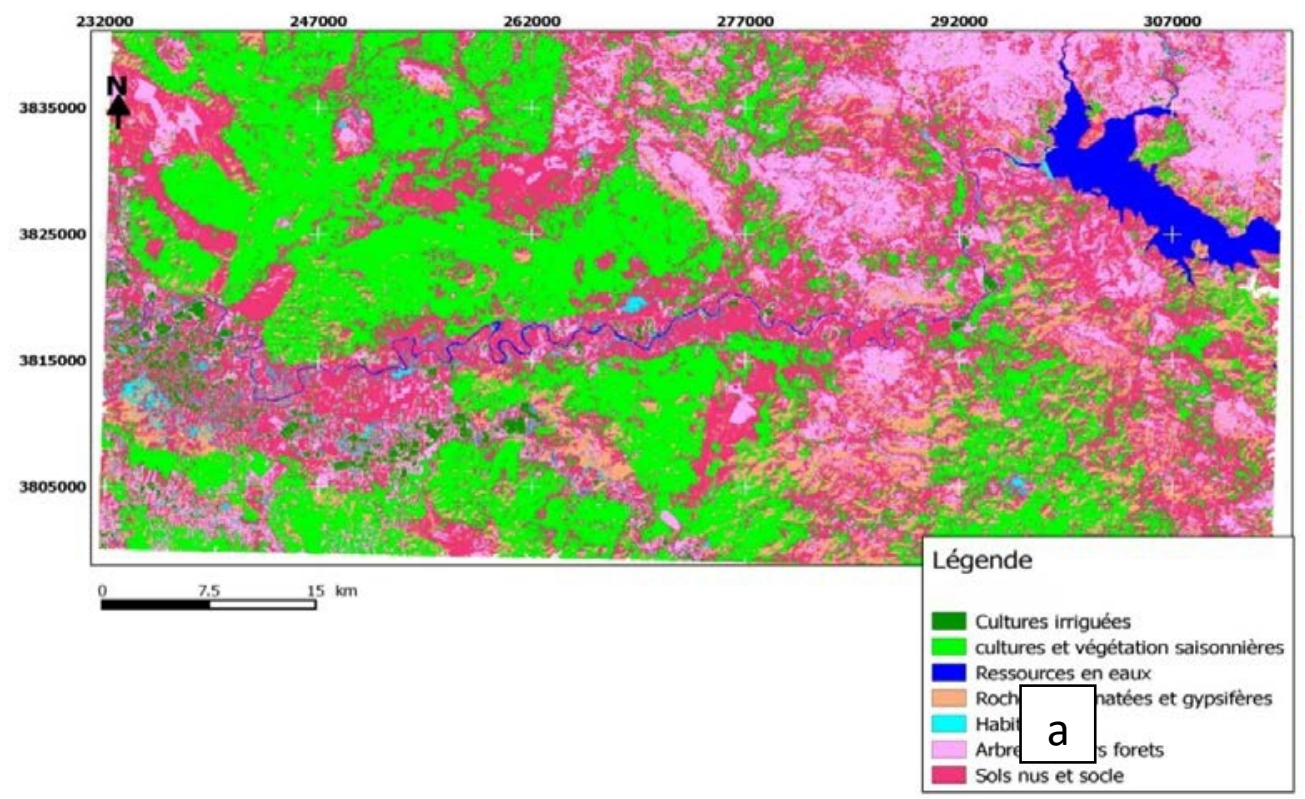



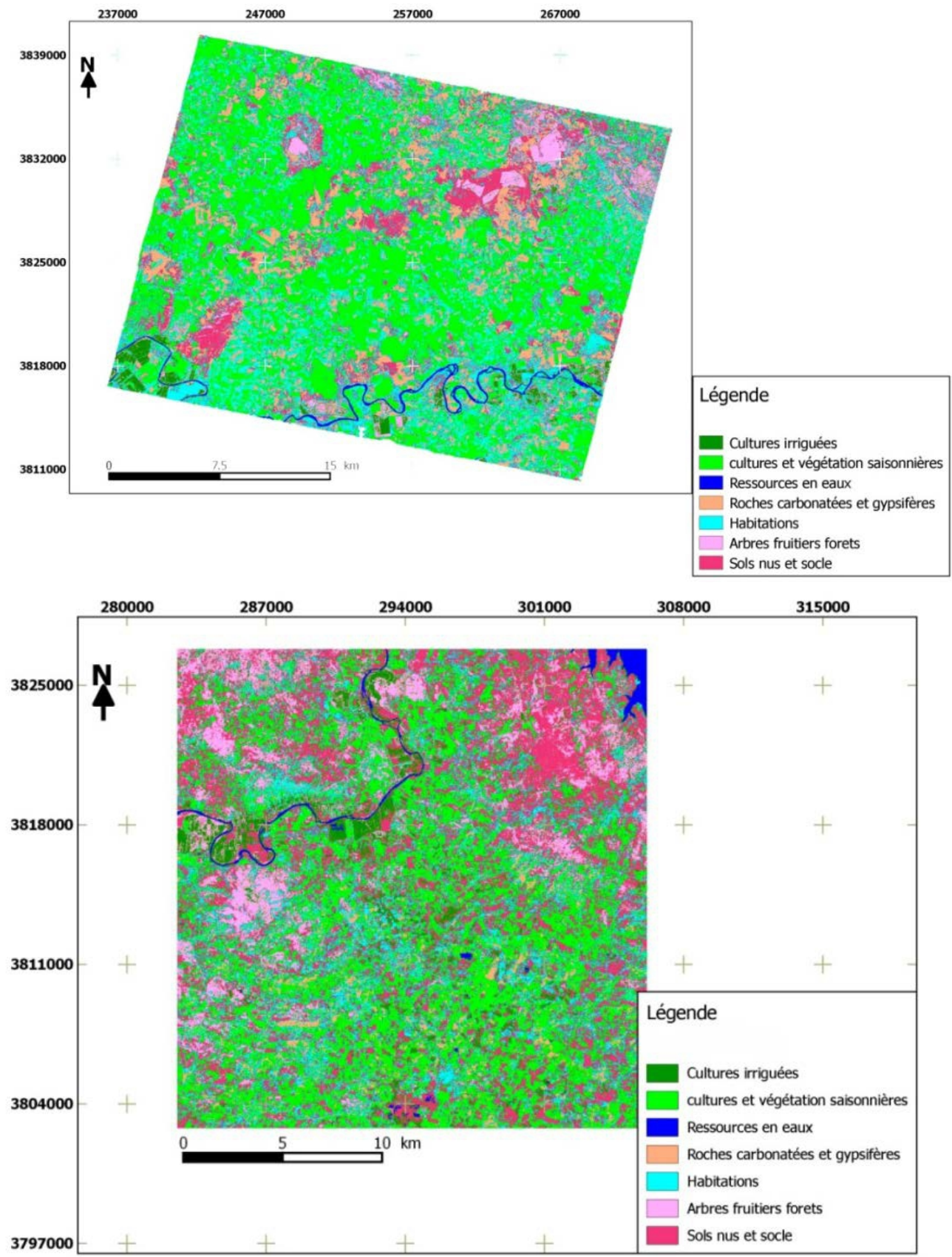

Figure 8: Résultats de la classification supervisée de type maximum vraisemblance sur les images (a) Landsat TM, (b) ASTER amont et (c) ASTER aval. 


\begin{tabular}{|c|c|c|c|c|c|}
\hline Image & Classes & Nature & Couleur & Superficie $\mathrm{m}^{2}$ & Pourcentage \% \\
\hline \multirow{8}{*}{$\begin{array}{l}\text { Landsat TM } \\
2009\end{array}$} & Classe 1 & Cultures irriguée & Vert foncé & 123161400.0000 & 4.835 \\
\hline & Classe 2 & $\begin{array}{l}\text { Culture bour ou } \\
\text { annuelle }\end{array}$ & Vert claire & 925167600.0000 & 36.317 \\
\hline & Classe 3 & Ressources en eaux & Bleu & 119133900.0000 & 4.677 \\
\hline & Class e 4 & $\begin{array}{c}\text { Roches carbonatées et } \\
\text { gypse }\end{array}$ & Marron claire & 317544987.0527 & 12.466 \\
\hline & Classe 5 & Habitations & Cyan & 64400800.0000 & 2.528 \\
\hline & Classe 6 & Arbres et forêts & Rose & 417993800.0000 & 16.591 \\
\hline & Classe 7 & Sols nus & Magenta & 579992123.3919 & 22.769 \\
\hline & Non classée & néant & néant & 0.0 & 0.0 \\
\hline \multirow{8}{*}{$\begin{array}{l}\text { ASTER } \\
\text { AMONT }\end{array}$} & Classe 1 & Cultures irriguées & Vert foncé & 45367150.9995 & 7.991 \\
\hline & Classe 2 & $\begin{array}{c}\text { Cultures et végétations } \\
\text { saisonnières }\end{array}$ & Vert claire & 165979630.2322 & 29.235 \\
\hline & Classe 3 & Ressources en eau & Bleu & 6783673.8968 & 1.195 \\
\hline & Class e 4 & $\begin{array}{c}\text { Roches carbonatées et } \\
\text { gypse }\end{array}$ & Marron claire & 23412120.0494 & 4.124 \\
\hline & Classe 5 & Habitations & Cyan & 80113881.0200 & 14.111 \\
\hline & Classe 6 & Arbres et forêts & Rose & 62693636.1418 & 11.043 \\
\hline & Classe 7 & Sols nus & Magenta & 183392473.0259 & 32.302 \\
\hline & Non classée & néant & néant & 0.0 & 0.0 \\
\hline \multirow{8}{*}{$\begin{array}{l}\text { ASTER } \\
\text { AVAL }\end{array}$} & Classe 1 & Cultures irriguées & Vert foncé & 23806593.5400 & 2.055 \\
\hline & Classe 2 & $\begin{array}{c}\text { Cultures et végétations } \\
\text { saisonnières }\end{array}$ & Vert claire & 664171603.1214 & 57.345 \\
\hline & Classe 3 & Ressources en eau & Bleu & 7486014.1504 & 0.646 \\
\hline & Class e 4 & $\begin{array}{c}\text { Roches carbonatées et } \\
\text { gypse }\end{array}$ & Marron claire & 118880625.4129 & 10.264 \\
\hline & Classe 5 & Habitations & Cyan & 207604344.1558 & 17.925 \\
\hline & Classe 6 & Arbres et forêts & Rose & 35396193.9389 & 3.056 \\
\hline & Classe 7 & Sols nus & Magenta & 100865828.1400 & 8.709 \\
\hline & Non classée & néant & néant & 0.0 & 0.0 \\
\hline
\end{tabular}

Tableau 2 : Statistiques des classes à partir de la classification supervisée de Maximum

Vraisemblance :

\section{Analyse des indices}

Les données radiométriques peuvent être utilisées sous forme de canaux bruts ou d'indices combinant les canaux rouge et proche infrarouge.

Il existe de nombreux indices du sol et de végétation déduits à partir des mesures de réflectance ou de comptes numériques pour estimer le taux de recouvrement et identifier les types des sols et du couvert végétal. Ces différents indices exploitent essentiellement la différence de réponse spectrale de la végétation et des sols dans la bande rouge $(\mathrm{R})$ qui est liée à l'absorption de la lumière par la chlorophylle et la bande proche infrarouge (PIR) qui est liée à la densité de la végétation verte.

Ces indices sont mathématiquement exprimés de manière à utiliser le contraste important entre ces deux bandes. Ce contraste résulte de la forte 
absorption du rayonnement dans le rouge et de la forte réflectance dans le proche infrarouge.

Apres avoir révélé la zone potentiellement affectées par la salinité à partir de la classification des images satellitaires Landsat et ASTER, nous avons pu utiliser les indices radiométriques suivants:

- SI : Indice de salinité

- NDSI : Indice de salinité par différence normalisée.

- NDVI : Indice de végétation par différence normalisée.

- $\quad$ BI : Indice de luminosité.

Les résultats de l'analyse des indices sur les images Landsat TM et ASTER sont représentés dans les figures 9 et 10 .

Les résultats de l'analyse des indices des données Landsat TM ont montré que les indices qui ont donné plus de résultats satisfaisants et qui ont pu distinguer les zones de salinité de celles non salines étaient les indices de NDSI et NDVI. Les indices SI et BI, quant à eux, ne montrent pas une grande différenciation entre les différentes classes représentées, et ne permettent pas vraiment une révélation des zones de salinité. En revanche, ils indiquent des petites zones qui présentent un grand indice de salinité, surtout là où on assiste à des affleurements des roches évaporitiques. Les autres formes et niveaux de salinité ne sont pas représentés dans les résultats de ce type d'indices.

Les résultats de l'analyse des indices des données Landsat TM peuvent être résumés comme suit:

- L’indice NDSI est un indice fiable pour déterminer les sources potentielles de salinité à la surface du sol. Il permet une grande distinction entre les différentes classes de la région surtout celle qui présente une source de salinité de celles qui ne le sont pas (Fig. 9B). Il permet de localiser les zones de forte salinité dans toute la région d'étude, chose qui n'est pas possible avec les autres indices.

- L'image de NDVI permet de différencier entre trois classes bien distinctes. Et cela pourrait aider à localiser les potentielles sources de salinité dans le cadre de notre étude (Fig. 9C). Toutefois, cette méthode ne pourrait pas différencier les zones affectées par la salinité de celles de la classe des sols nus surtout qui sont situées dans les régions proche de la rivière ou celles de moyenne altitude dans le cas de notre étude. Cette image NDVI pourrait également ne pas discriminer les plans d'eau des zones humides.

- Les images de l'indice de salinité (SI) et l'indice de luminosité (BI) des données Landsat TM sont présentées dans les figures 9A et 9D respectivement. Ces indices ont montré une grande potentialité dans la localisation des niveaux élevés de salinité. Toutefois, leurs 
résultats ne peuvent pas distinguer entre les zones salines et les zones non salines comme c'est le cas pour les images NDSI et NDVI.

Les résultats de l'analyse des images des indices calculés sur la base des données des images ASTER peuvent être résumés comme suit:

- Les images des indices NDSI et NDVI dérivées et générées à partir des données ASTER donnent des résultats plus satisfaisants dans la différenciation entres les zones salines et les zones non salines dans les parties choisies de notre région d'étude (Fig. 10). Elles

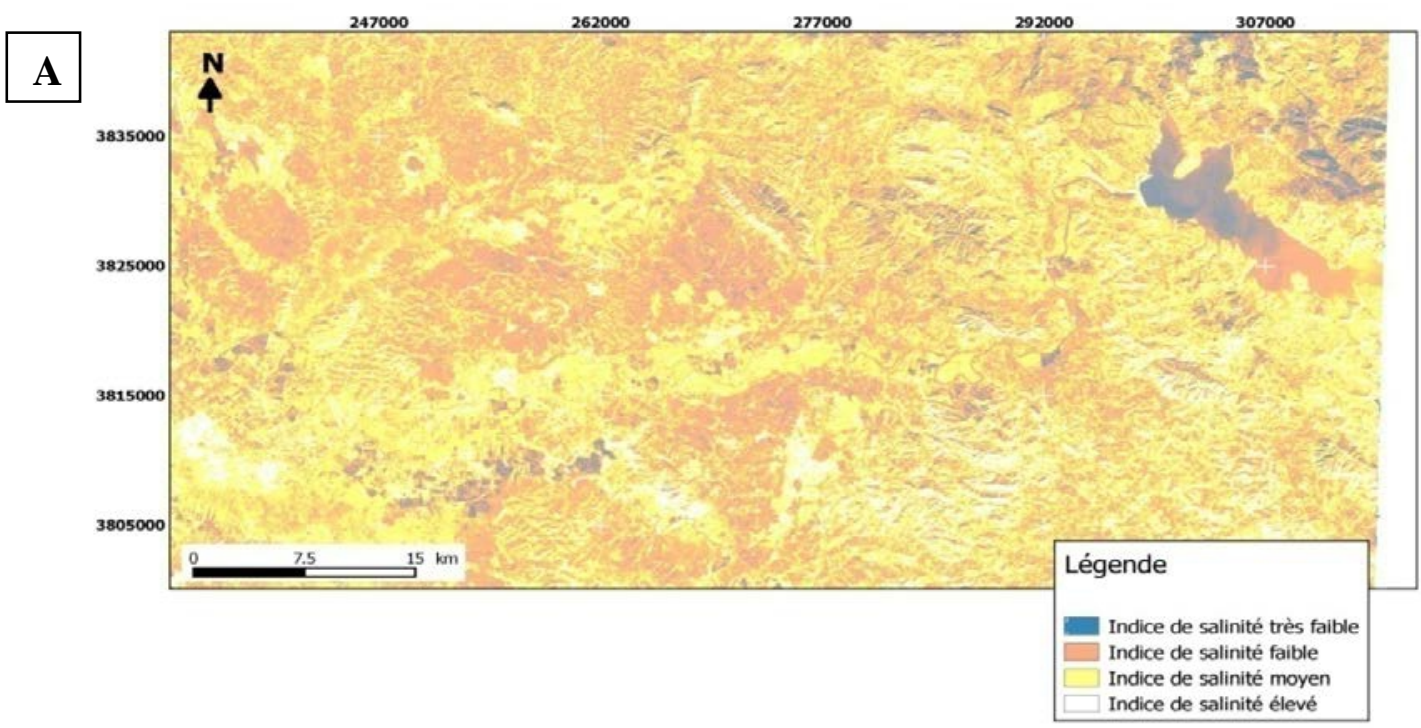

B

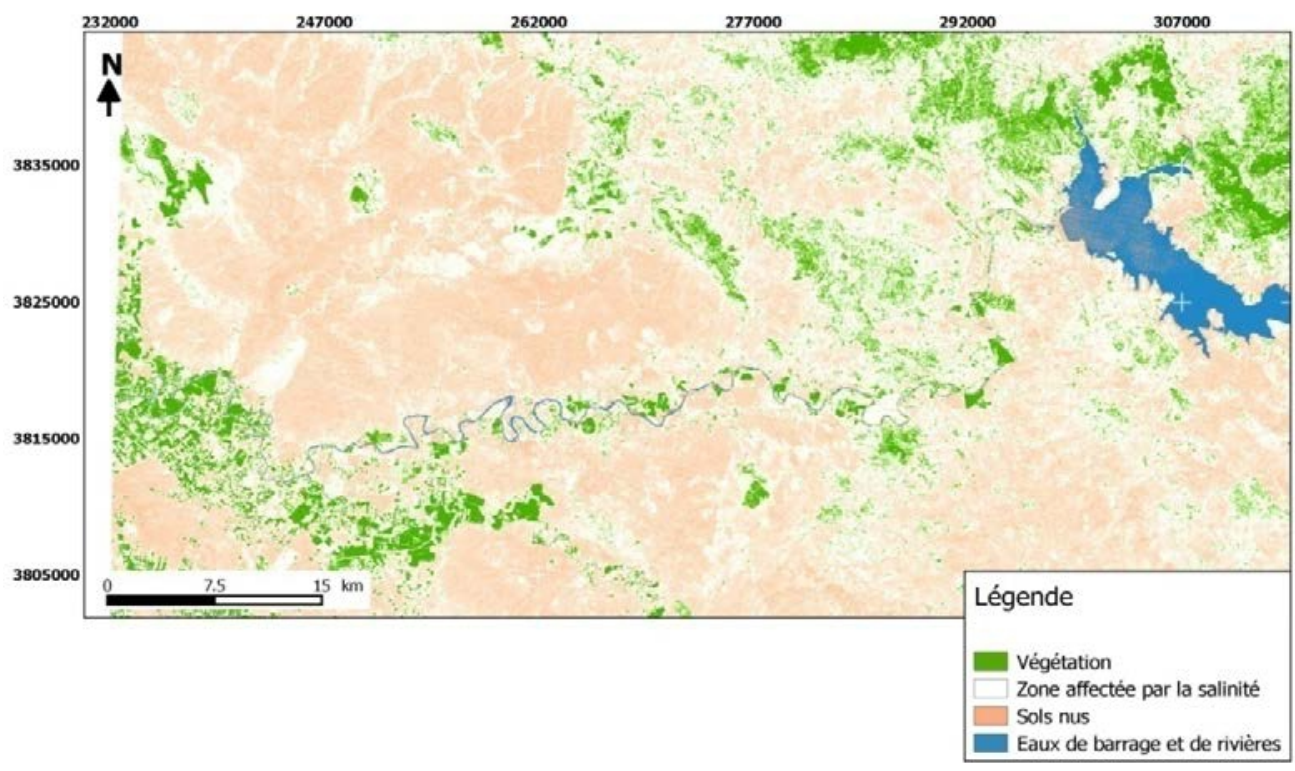



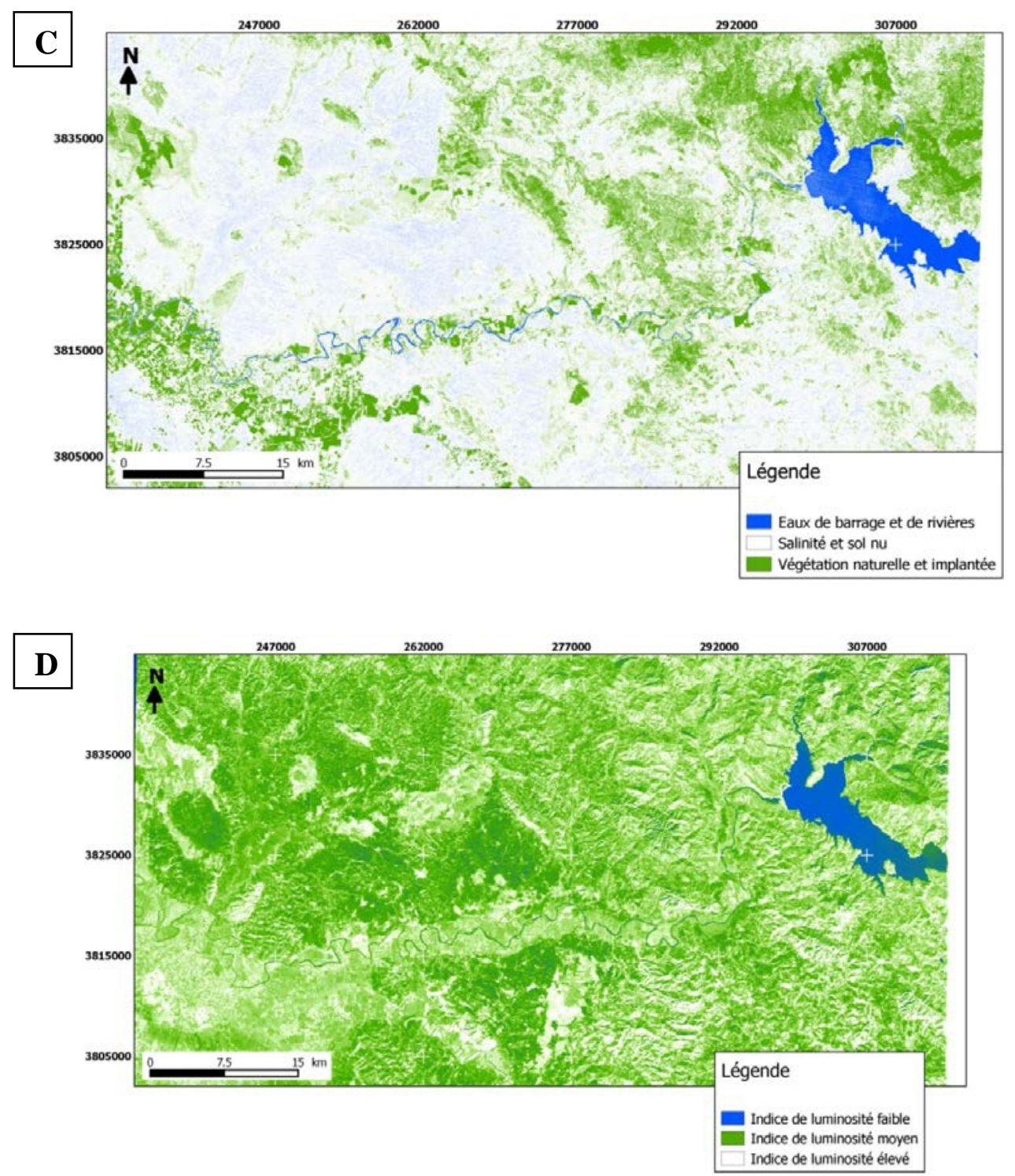

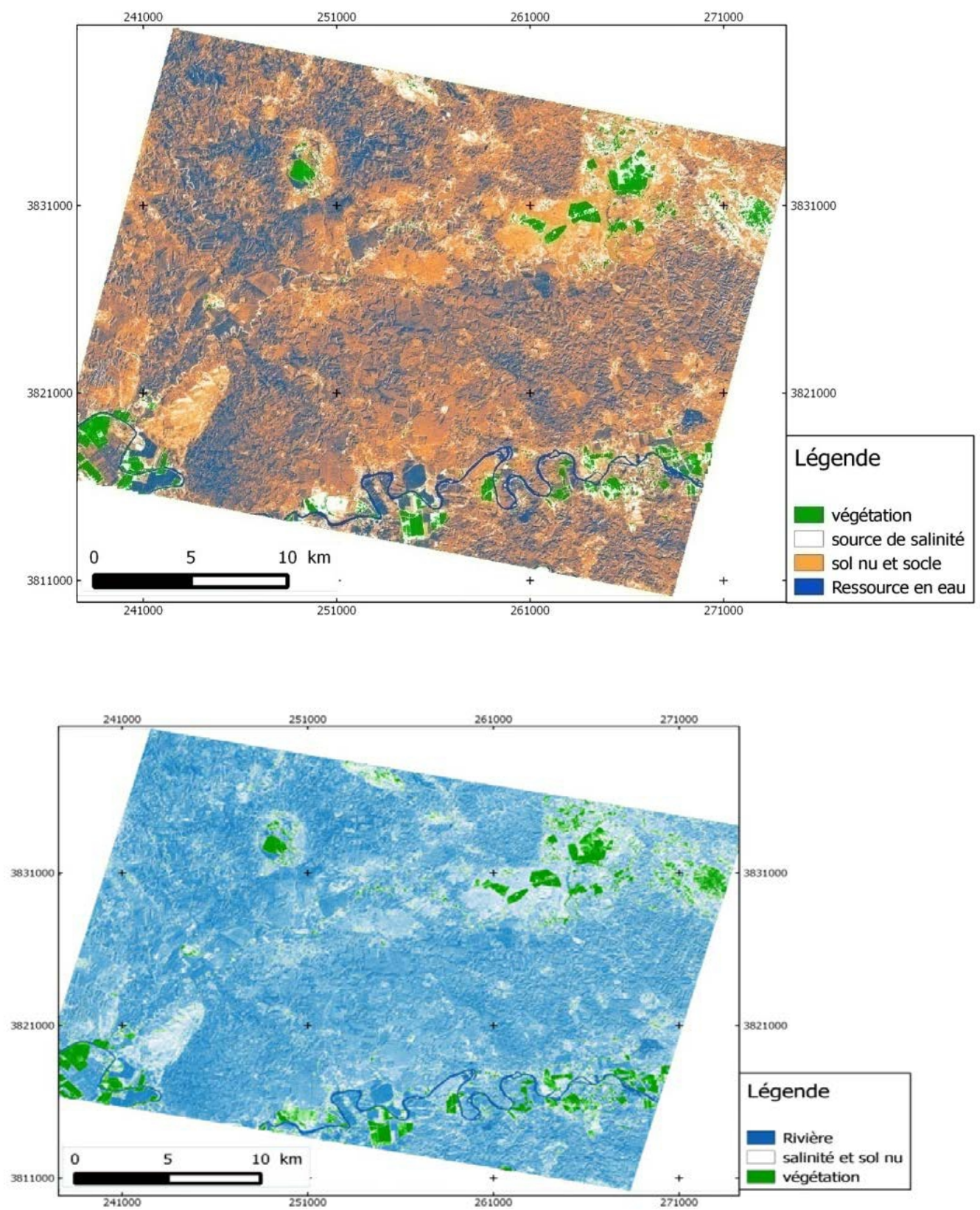

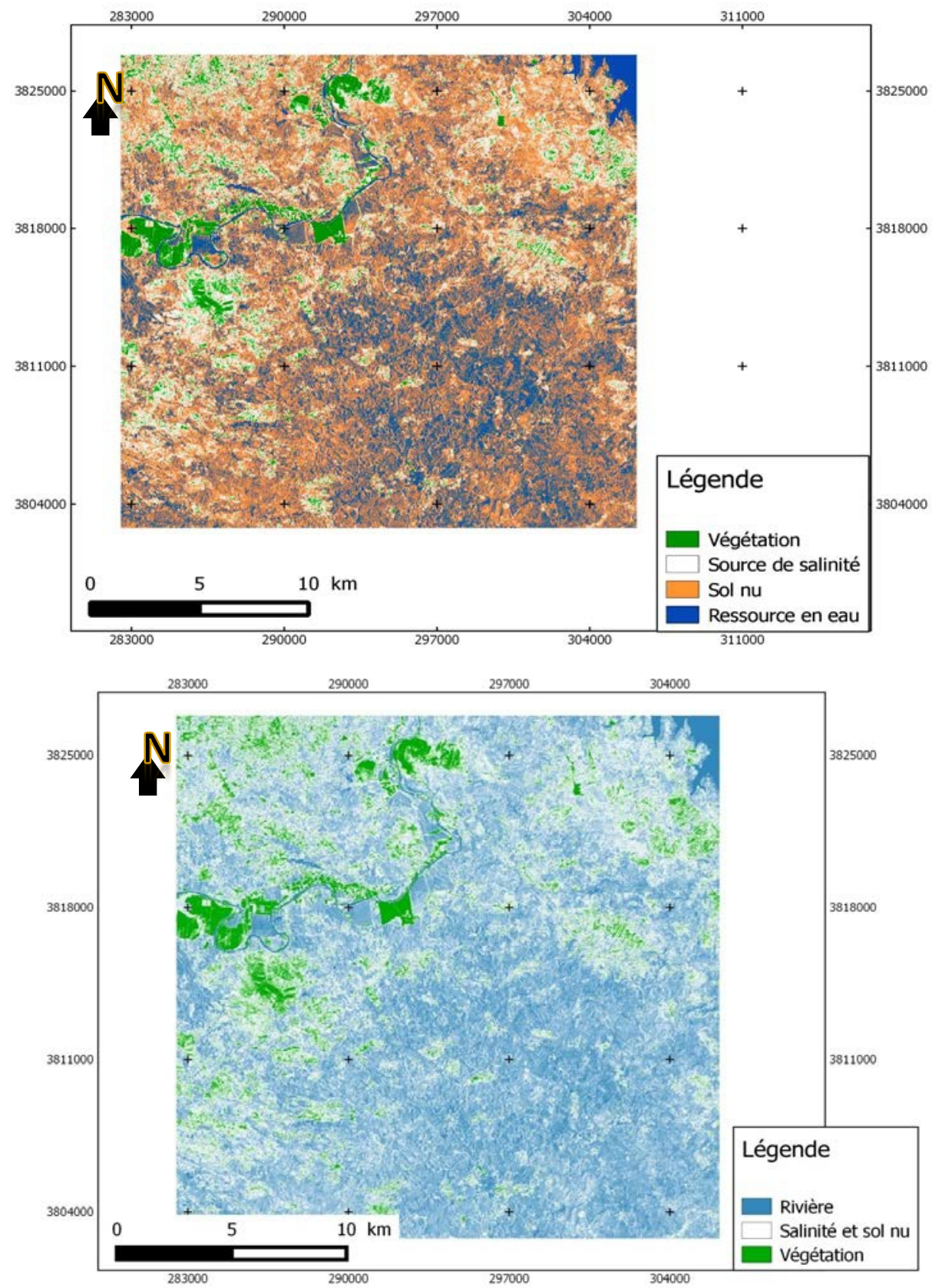

Figure 10: Cartes de la salinité, à la surface du sol de la région de l’Ouergha, dérivée à partir du calcul de l'indice NDSI (en haut) et l'indice NDVI (en bas) sur l'image ASTER amont (à droite) et aval (à gauche). 
permettent plus de précision pour la détection des sources de salinité à la surface du sol. Par conséquent, ces données pourraient être un outil complémentaire à l'étude par les images Landsat, puisqu’elles permettent d’offrir plus de distinction et de localisation entre les classes de la région.

- Sur les résultats des indices NDSI calculés à partir des images ASTER, les sources de salinité et/ou celles qui pourraient constituer des sources potentielles sont facilement repérables par rapport aux zones de non salinité. L'application de cet indice aux données ASTER pourrait devenir une source fiable de classification, cela permet même de différencier les différents niveaux de salinité que pourraient avoir une région qui présente les mêmes caractéristiques environnementales que la nôtre.

- En outre, les plans d'eau pourraient être plus précisément différenciés des zones d'humidité élevés que ceux des images Landsat.

- Cependant, l'image NDVI dérivées des données ASTER ne pourrait pas différencier entre les sols nus et les zones affectées par la salinité.

- Par conséquent, il est très utile pour les travaux futurs d'appliquer cette méthode à d'autres scènes ASTER afin de détecter et de localiser, avec une plus grande précision, les zones affectées par la salinité dans la région de l’Ouergha.

Distribution de la salinité dans la région d’étude

Conformément à l'objectif de l'étude, la démarche de travail est fondée sur l'identification puis la délimitation des unités des sols particulièrement les formations gypseuses, obtenues à l'aide des réflectances exo-atmosphériques, puis, par des classifications non supervisées et supervisées sur une trichromie TM et ASTER brut $(R=B 1, V=B 2, B=B 3)$. Et par la suite les comparer avec les résultats de l'analyse d'eaux souterraines pour pouvoir comprendre l’origine de la salinité des eaux.

D’après l'étude géologique et hydrochimique, les évaporites constituent une part importante de la lithologie de la zone d'étude (Fig.1B). Leur intensité varie dans la zone d'étude suivant la l'altitude ainsi que dans l'espace. Cette situation joue un rôle important en termes de niveau de salinité et de sa distribution.

\section{Distribution suivant le niveau de salinité}

A partir de l'interprétation visuelle des résultats du traitement de l'image Landsat TM et ASTER, couplé par l'étude de terrain, nous avons pu distinguer trois niveau de salinité dans la zone d’étude (Figure 11) :

- $\quad$ Niveau de salinité élevé qui se manifeste par une couleur blanche sur l'image.

- $\quad$ Niveau de salinité moyen qui se manifeste par une couleur claire du bleu et/ou du gris sur l'image. 
- Et niveau de salinité bas marquée par les couleurs rouge, rose, orange et ver.

\section{Distribution suivant la topographie et la géologie}

La salinité dans la zone d'étude peut également être classifiée en deux catégories relativement liées aux conditions de la topographie et de la géologie, ainsi nous pouvons avoir:

- Une Salinité qui prend son origine des reliefs surtout des nappes de charriage.

- Et une salinité liée aux mécanismes de l’hydrologie locale (Rivières). Le diagramme conceptuel montrant la relation entre la salinité dans le secteur d'étude et la topo-géologie est schématisé dans la figure 12.

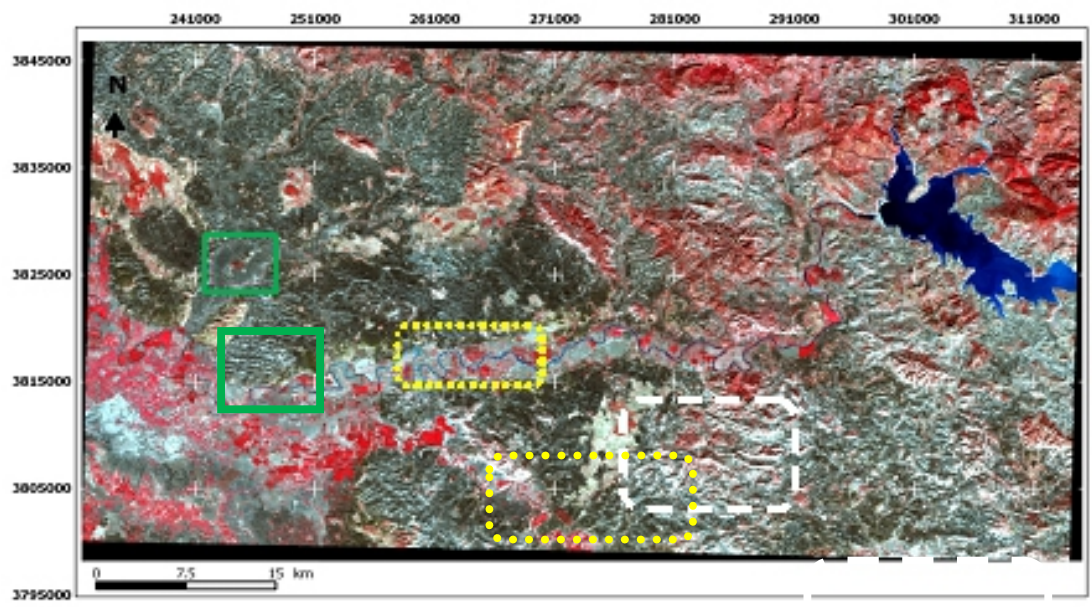

Figure 11 : Composition colorée à partir de l'image TM 2009 (RGB $\angle 32)$ montre les diff '́rents niveaux de salinité dans la zone d'étude. Une salinité élevée (rectangle blanc), une salinité (rectangle jaune), et une salinité faible (rectangle vert).

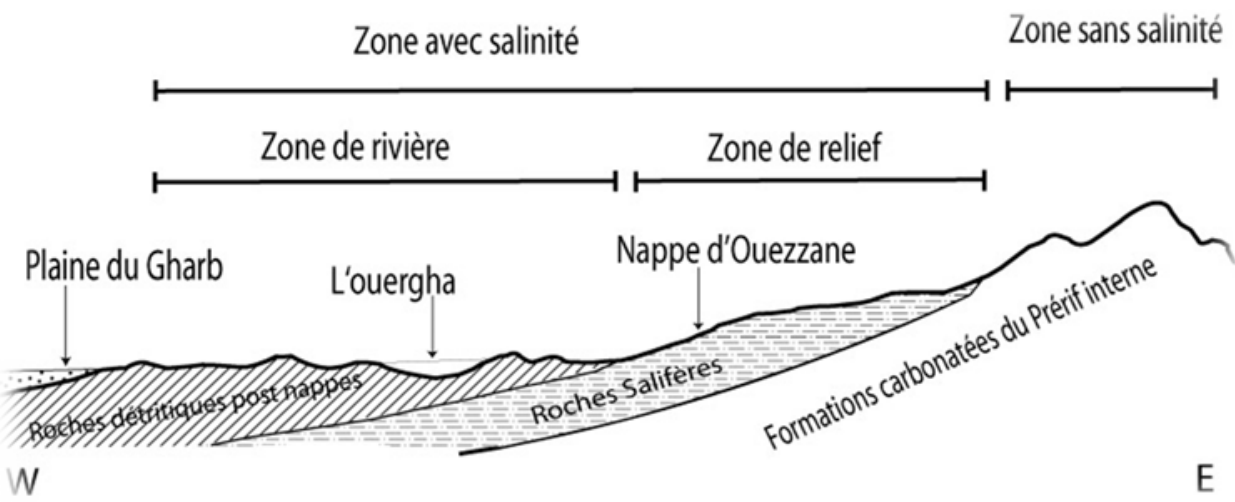




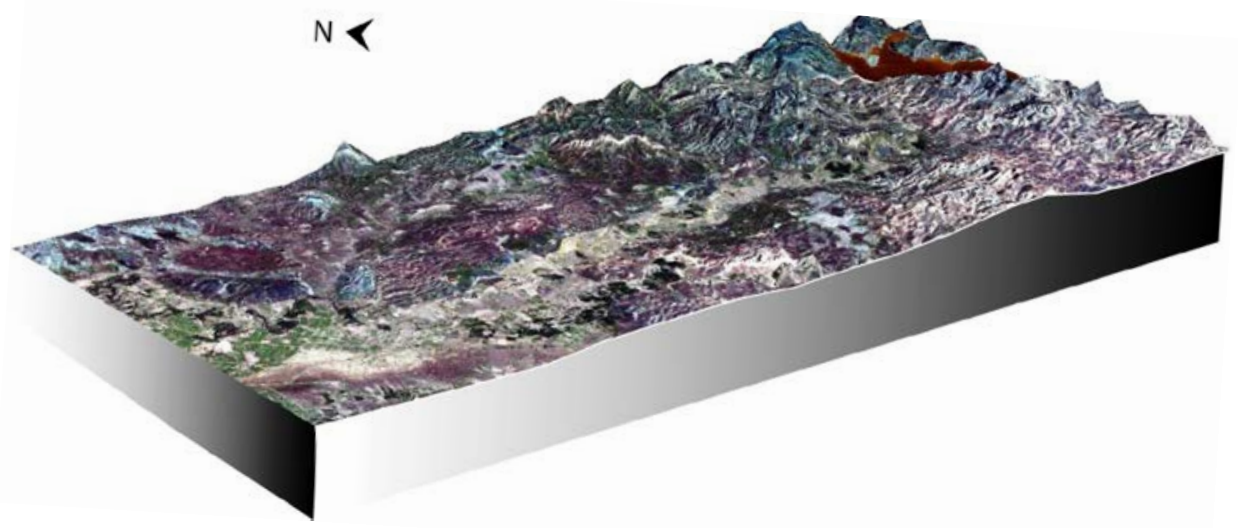

Figure 12 : Schémas du diagramme conceptuel de la relation entre la salinité dans la zone et la géologie de la région.

\section{Conclusion}

Dans cette étude nous avons utilisé les potentialités offertes par les données de télédétection pour cartographier les zones de salinité à la surface du sol dans la région de l'Ouergha.

Les résultats achevés montrent qu'il est possible de réaliser une cartographie de la surface des sols dans la région de l'Ouergha à partir du traitement et de l'analyse des images satellitaires Landsat TM et ASTER. Ainsi, l'étude des résultats de ces traitements a permis de cartographier les zones qui constituent un risque de salinisation de sol et de l'eau et celles qui ne le sont pas. L'évaluation des résultats de l'application de cette méthode sur l'image Landsat TM a donné une précision de 87 pour cent. Toutefois, il est possible d'améliorer la précision et la qualité des résultats en utilisant des images ASTER comme données de bases à traiter. La résolution spatiale nécessaire pour cartographier la salinité à la surface du sol est de donc de 15 $\mathrm{m}$ à $30 \mathrm{~m}$.

La méthode de la classification supervisée de maximum vraisemblance appliquée sur les données satellitaires disponibles a montré sept classes de l'occupation du sol dans la région de l'Ouergha. Cette classification donne un pourcentage élevé des zones susceptibles d'être une potentielle source de salinité surtout avec les images ASTER qui sont choisis sur la base des résultats précédents.

L'étude par les indices radiométriques choisis dans le cadre de cette étude, pour soutenir la méthode supervisée, a montré des résultats satisfaisants, et confirme les résultats de la classification, surtout avec l'indice NDSI et NDVI qui ont permis une parfaite distinction entre les zones de salinité et les zones de végétation. Ainsi, cette étude nous a permis 
également de mettre en évidence une relation inverse entre la salinité et le couvert végétal dans la région étudiée.

Cette étude démontre encore une fois l'origine naturelle, liée à la lithologie de type évaporitique, de la salinité des eaux souterraines de la région étudiée.

Les compétences développées dans cette étude fournissent une bonne base pour développer davantage cette technique afin de pouvoir l'appliquer à l'échelle régionale pour cartographier tous les aspects possibles de la salinité à la surface des sols pour plus de maîtrise de la gestion des ressources naturelles de la région.

\section{References:}

Abbas, A., Khan, S., Hussain, N., Hanjra, M. A., \& Akbar, S. (2013). Characterizing soil salinity in irrigated agriculture using a remote sensing approach. Physics and Chemistry of the Earth, Parts A/B/C, 55, 43-52.

Abourida A., (2007). Approche hydrogeologique de la nappe du haouz (Maroc) par teledetection, isotope, SIG et modélisation. Thèse de doctorat, Faculté des Sciences Semlalia Marrakech. 146 pages.

Al Khair F., (2003); Soil salinity detection using remote sensing. Thesis submitted to international institute for Geo-Information science and earth observation in partial fulfillment of the requirements of the degree of Master of Science in Geo-information science and earth observation, watershed management, conservation and river basin planning specialization. 70p.

Bishop, T. F. A., \& McBratney, A. B. (2001). A comparison of prediction methods for the creation of field-extent soil property maps. Geoderma, 103(1), 149-160.

Bonn F., charbonneau L. \& Brochu R. (1989). Analysede l'utilisation des sols à partir des données SPOT et TM : aspects méthodologiques. Télédétection en francophonie. Ed. aupelf-uref. John libbey Eurotext. Paris. P. 83-92.

Boulahouat, N., \& Naert, B. (1996). Télédétection des ressources en sols des zones arides. Revue étude et Gestion des sols, 7-26.

Carré, F., \& Girard, M. C. (2002). Quantitative mapping of soil types based on regression kriging of taxonomic distances with landform and land cover attributes. Geoderma, 110(3), 241-263.

Dib I., Boudoukha A. et Chettah W., (2010). L’impact de l'activité agricole et urbaine sur la qualité des eaux souterraines de la plaine de Gadaine - Ain Yaghout (Batna est-algérien).

Douaoui, Abd El Kader, Hervé Nicolas, and Christian Walter, (2006). "Detecting Salinity Hazards within a Semiarid Context by Means of Combining Soil and Remote-Sensing Data.” Geoderma 134 (1-2): 217-30. 
Durand Delga M. \& al., (1961): Domaine rifain : carte de la géologie structurale du domaine rifain.

Abbas, A., Khan, S., (2007). Using remote sensing technology for appraisal of irrigated soil salinity. In: Oxley, L., Kulasiri, D. (Eds.), MODSIM 2007 International Congress on Modelling and Simulation. Modelling and Simulation Society of Australia and New Zealand, pp. 2632-2638.

Dwivedi, R., (1992). Monitoring and the study of the effects of image scale on delineation of salt-affected soils in the Indo-Gangetic plains. International Journal of Remote Sensing, 13(8), 1527-1536.;

Escadafal, R., Girard, M. C., \& Courault, D., (1988). Modeling the relationships between Munsell soil color and soil spectral properties. International agrophysics, 4(3), 249-261.

Escadafal, R., Girard, M. C., \& Courault, D., (1989). Munsell soil color and soil reflectance in the visible spectral bands of Landsat MSS and TM data. Remote Sensing of Environment, 27(1), 37-46.

Escadafal, R., (1993). Remote sensing of soil color: principles and applications. Remote Sensing Reviews, 7(3-4), 261-279.

Escadafal, R., (1994). Soil spectral properties and their relationships with environmental parameters-examples from arid regions. In Imaging Spectrometry-A Tool for Environmental Observations (pp. 71-87). Springer Netherlands.

Girard M-C., (1983). Télédétection de la surface du sol. Application de la télédétection à l'agriculture. Coll. INRA. N 32, pp 177-193.

Girard, C. M., Gilliot, J. M., Girard, M. C., \& Thorette, J., (1997). Comparaison de la cartographie de l'occupation des terres par classification de données de télédétection avec la cartographie CORINE niveau 3: application à une zone au nord-ouest de l'Île de France. Revue internationale de géomatique, 7(1), 57-86.;

Girard mc, girard c.m., (1999). Traitement des données de télédétection.

DUNOD Ed. Paris, 529 p.

Hamdi Aissa, B. (2001). Le fonctionnement actuel et passé de sols du Nord Sahara (cuvette de Ouargla) Approches micromorphologiques, géochimique, minéralogique et organisation spatiale (Doctoral dissertation).

Khan, N. M., Rastoskuev, V. V., Sato, Y., \& Shiozawa, S. (2005). Assessment of hydrosaline land degradation by using a simple approach of remote sensing indicators. Agricultural Water Management, 77(1), 96-109.

Metternicht, G., \& Zinck, J. A. (1997). Spatial discrimination of salt-and sodium-affected soil surfaces. International Journal of Remote Sensing, 18(12), 2571-2586

Metternicht, G. I., \& Zinck, J. A. (1998). Evaluating the information content of JERS-1 SAR and Landsat TM data for discrimination of soil erosion 
features.ISPRS Journal of Photogrammetry and Remote Sensing, 53(3), 143153.

Metternicht, G. I., \& Zinck, J. A. (2003). Remote sensing of soil salinity: potentials and constraints. Remote sensing of Environment, 85(1), 1-20.

Mougenot, B. (1993). Effets des sels sur la réflectance et télédétection des sols salés. Cahiers ORSTOM, Serie Pedologie, 28, 45-54.

Piquee A., Soulaimani A., Hoepffner Ch., Bouabdelli M., Laville E., Amrhar M et Chalouan A. 2007. Géologie du Maroc, édition GEODE, collection terre et patrimoine, Série Manuels 287 pages. Le Rif externe p 188.

Rahman, H., \& Dedieu, G. (1994). SMAC: a simplified method for the atmospheric correction of satellite measurements in the solar spectrum.Remote Sensing, 15(1), 123-143.

Richards, J. A., \& J. Xiuping (1999). Remote sensing digital image analysis (Vol. 3). Springer New York.

Sadiki M. Et Mrabet S. (2015) apport de la spatialisation des données a la caractérisation physico-chimique des ressources en eau. Cas du bassin de l'Ouergha. Maroc. Arabian journal of earth sciences, vol.4 (2015) - issue 1: 1-23.

Schowengerdt, 2007. R.A. Schowengerdt. Remote sensing: Models and methods for image processing (3rd ed.)Elsevier Inc., USA (2007) p. 515.

Srivastava, A., Tripathi, N. K., \& Gokhale, K. V. G. K. (1997). Mapping groundwater salinity using IRS-1B LISS II data and GIS techniques.International Journal of Remote Sensing, 18(13), 2853-2862.

Thauvin J.P. (1971). Ressources en eau du Maroc tome 1. Domaines du rif et du Maroc oriental éditions du service géologique du Maroc rabat 1971, 313p. Pages 31; 32.

USGS, and Japan ASTER Program (2003), ASTER scene AST_L1B_003_06262000100635,1B, USGS, Sioux Falls, 6/26/2003.

Wannakomol, A. (2005). Soil and Groundwater Salinization Problems in the Khorat Plateau, NE Thailand (Doctoral dissertation, Freie Universität Berlin, Germany). 